\title{
DER ZOOLOGISCHE GARTEN
}

ZEITSCHRIFT FUR DIE GESAMTE TIERGARTNEREI

\author{
ORGAN DER KOMMISSION FUR TIERGARTEN \\ DER DEUTSCHEN DEMOKRATISCHEN REPUBLIK, \\ DES VERBANDES DEUTSCHER ZOODIREKTOREN \\ UND DES INTERNATIONALEN VERBANDES \\ VON DIREKTOREN ZOOLOGISCHER GARTEN
}

BEGRUNDET VON

GEORG GRIMPE

FORTGEFUHRT VON

KARL MAX SCHNEIDER

HERAUSGEGEBEN VON

Prof. Dr. Dr. HEINRICH DATHE

(NEUE FOLGE)

46. BAND $\cdot 1976$

\section{VEB GUSTAV FISCHER VERLAG JENA}




\section{Inhaltsverzeichnis von Band 46}

\section{(Seiten 1-20s: Dathe-Festschrift II)}

(Die mit * bezeichneten Abhandlungen sind bebildert.)

Sicifert, S.: Heinrich Dathe zur Vollendung seines 65. Lebensjahres . . . . . . . . 1

*Rawlins, C. G. C.: Reginald Greed $\dagger \ldots \ldots \ldots \ldots \ldots \ldots \ldots \ldots \ldots \ldots \ldots \ldots \ldots \ldots \ldots$

\section{Abhandlungen}

*Altmann, D., u. Scheel, H.: Ethologische Studien an Riesenelenantilopen, Trurotragus derbianus gigas Heuglin (Mammalia, Artiodactyla) .............. 118

*Asanow, N. S., u. Lippert, W.: Bemerkungen zum Verhalten einer Mantelpaviangruppe (Papio hamadryas) gegenüber einer Mutter mit Zwillingen . . . . . . . . 108

*Bock, H.-J., u. Haas, G.: Natürliche und künstliche Aufzucht von Saruskranichen

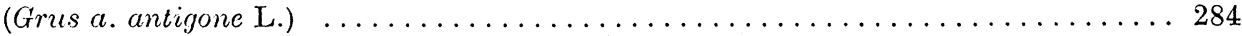

*Briedermann, L.: Ergebnisse einer Inhaltsanalyse von 665 Wildschweinmagen ... 157

*Bürger, M.: Ein Haus für Elefanten, Flußpferde und Nashörner im Zoologischen Gar-

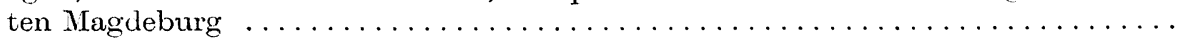

*Busse, E., s. Busse, H.

*Busse, H. u. E.: Erfolgreiche künstliche Aufzucht von Schnee-Eulen (Nyctea scandiaca

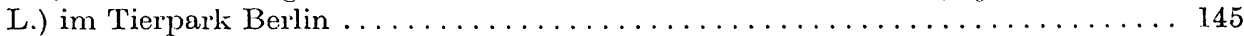

*Cousins, D.: 'The Breeding of Gorillas, Gorilla gorilla, in Zoological Collections ... 215

*Dathe, H. H., s. Dathe, R.

*Dathe, R., Dathe, H. H., u. Nagel, R.: Beobachtungen zur Mutter-Kind-Beziehung

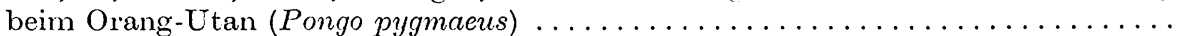

Fíbián,L.: Darmpech-Obstipation (,Fohlenkolik") bei neugeborenem Zwergflußpferd,

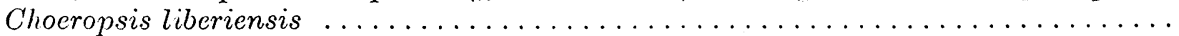

*Faust, R., s. Hoffmann, R.

Forstner, M. J., Wiesner, H., Jonas, D., u. Kraneburg, W.: Versuche zur Entwurmung von Zoowiederkäuern und -equiden mit Mebendazol . .......... 401

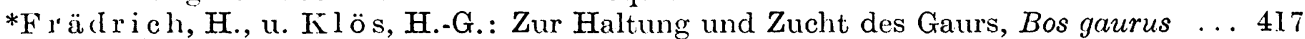

*Gehring, C. B.: Grußverhalten und Erkennen vertrauter Personen sowic weitere Verhaltensweisen einer Kapuzinergruppe $($ Cebus apella $)$ im Zoo . . . . . . . . . . 353

Gorgas, M.: Über ein zerebrales Larva migrans visceralis-Syndrom und seine Behand-

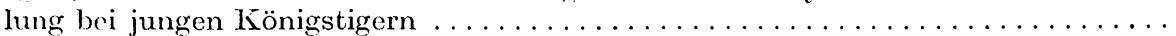

*Grummt, W.: Beitrag zur Fortpflanzungsbiologic des Kuckucksikauzes, Ninox novae-

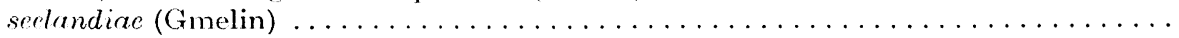

*Haas, G., s. Bock, H.-J.

*Hagen beck, C. C.: Haltung und Zucht von Mandschuren-Kranichen, Grus japonensis, in Carl Hagenbecks Tierpark, Hamburg-Stellingen . . . . . . . . . . . . 371

*IToffmann, R., Faust, R., Weinandt, U., u. Hoffmann-Fezer, G.: Chromosomenuntersuchungen an fünf Spezies der Ordnung Falconiformes . . . . . . . . . .

*Hoffmann-Fezer, G., s. Hoffmann, R.

*Jacob, K.-J.: Zur Haltung und Zucht der Schellente, Bucephala clangula (L.) . . . 139

Jonas, D., s. Forstner, M. J.

*Kirsche, W.: Beitrag zur Biologie der Sternschildkröte (Testudo elegans Schoepff) . .

*Klös, H.-G., s. Frädrich, H.

Kraneburg, W., s. Forstner, M. J.

*Lang, E. M.: Haltung und Zucht des Kleinen Kudu (Tragelaphus imberbis) . . . . . . . .

*I.ange, J.: Beitrag zur Haltung und Aufzucht von Königspinguinen, Aptenodytes

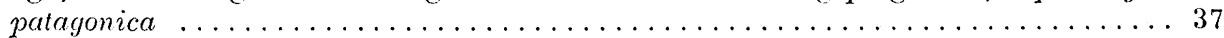

*Lau, D.: Reaktionen von Honigbienen (Apis mellifica L.) auf Polarisationsmuster an

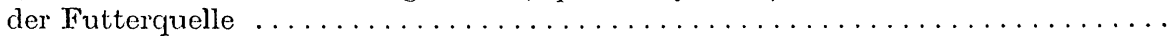

*Lippert, W., s. Asanow, N. S. 


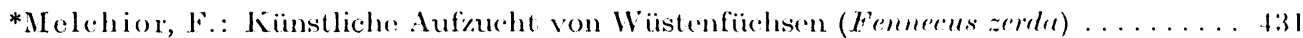

*Menzdorf, A.: Bemerkungen zur Siedlungsdichte und Reviergröße von steinhühnem,

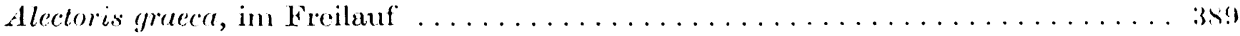

*Minnemann, D). Anfzucht cines Weißkopfsecallers (Halineetus leucocephalus) durch

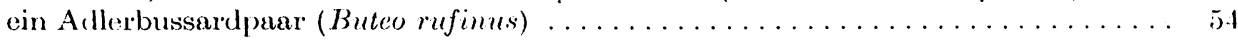

*Nagel, R., s. Dathe, R.

* Neugebauer, W.: Maul- und Klanenseuche bei Kiragenbären (Ursus thibetamus) . . 195

*Pechlaner, H.: Bastardierung von Alpenkrähe (Pyrrhocorax pyrrhocorax) und Alpen-

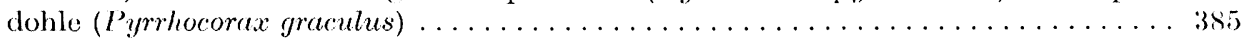

*Pelosse, J. L.: Etude sur le comportement d' attention che\% une population de chev-

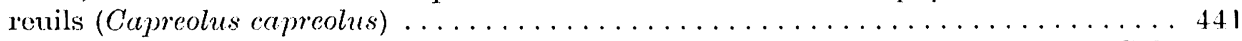

*1. et $\%$ old, H.-G.: Bemerkungen zur Fütterungstechnik bei nahmungsspezialisierten Gift nattern und einige Angaben zur Haltungsdauer von Elapiden im Tierpark Berlin . .

*Poglayen-Neuwall, Ingeborg, s. P'oglayen-Neuwall, I.

*Poglayen-Neuwall, I.: Zur Fortpflanzungsbiologie und Jugendentwicklung von

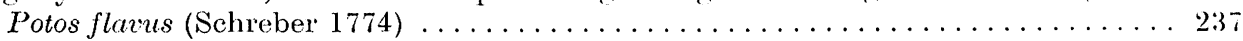

* _, - - and I. : Little known Mexican and Central American Zoos and Menageries . . . . . 186

*Psenner, H.: Haltung und Zucht des Bartgeiers (Gypaetus barbatus) . . . . . . . 293

Reuther, R. T.: Modern Zoo Administration ..................... 426

*Scheel, H., s. Altmann, D.

*Schürer, U.: Beobachtungen an einem neugeborenen Flachlandtapir, Tapirus terre-

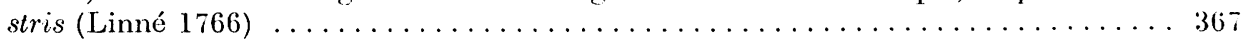

*Seitz, A.: Weitere Erhebungen zur Lebensdauer Asiatischer Elefanten, Elepha.*

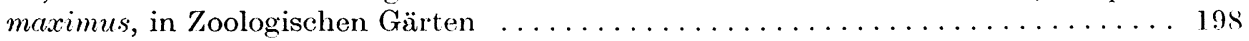

*Tscherner, W.: Eine neue Kokzidienart aus dem Tillipsenwasserbock (Kobus ellip)-

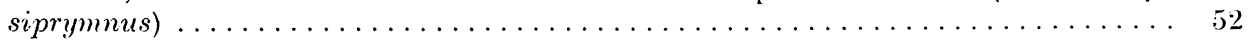

*Weinandt, U., s. Hoffmann, R.

*Wheater, R. J.: The Breeding of Gentoo Penguins, Pygoscelis papur, in Edinburgh

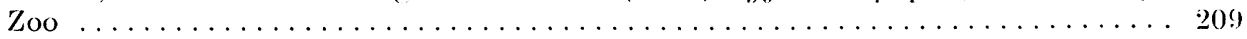

Wiesner, H., s. Forstner, M. J.

*W üst, G.: Geburt und perinatales Verhalten beim Steppenzebra, Equus quagga ... . 305

Berichte

Vergleichende Pathologie:

Therapie 453 


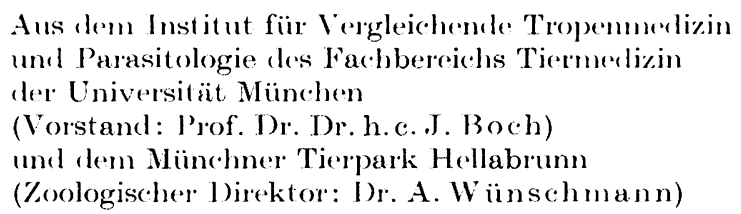

\section{Versuche zur Entwurmung von Zoowiederkäuern und -equiden mit Mebendazol}

Von Max Joachim Forstner, Henning Wiesner, München, Detlef Jonas, Koblenz, und Wilhelm Kraneburg, Miinchen

Zootiere sind in ganz besonderem Maße Wurminfektionen ausgesetzt. Dies hat seine Ursache einmal in der, von der Aufgabe eines Tierparks her, notwendigen dichten Besetzung gegebener Gehege, die bei der hohen Vermehrungsrate endoparasitischer Helminthen in kurzer Zeit mit ansteckungsfähigen Entwicklungsstadien übersät sind. Während die hier gehaltenen Tiere in der Weite ihres heimatlichen Lebensraumes nur selten wiederholten massiven Infektionen ausgesetzt sind, haben sie im Tierpark stets Gelegenheit zur Aufnahme parasitärer Keime (Enigk 1961, Boch 1961). Anders geartete klimat ische Verhältnisse sowie eine nicht immer dem Ursprungsland entsprechende Fütterung fördern den Ausbruch von klinischen Helminthosen, die besonders bei der Aufzucht von Jungtieren zu Verlusten führen können (Zuchowska 1969; Ippen u. Schröder 1970). Daß Streßsituationen durch soziale Rangfolgeauseinandersetzungen bei ungenïgender oder fehlender Fluchtmöglichkeit den Ausbruch parasitärer Erkrankungen zu begiinstigen vermögen, ist nicht von der Hand zu weisen, wie auch ähnliche Faktoren auf den Erfolg von Wurmkuren Einfluß haben (Mackay 1974).

Eine besondere Bedeutung bei der Bekämpfung des Wurmbefalls von Zootieren kommt der Prophylaxe zu (Lahde 1972). Hier sind in erster Linie Maßnahmen zu erwähnen, die eine Unterbrechung des parasitären Entwicklungszyklus gewährleisten. Neben der regelmäßigen Kotentfernung ist auf die Freiheit des Futters von Wurmeiern und -larven zu achten, Bodenfütterung also tunlichst zu vermeiden. Der die Entwicklung von Parasitenkeimen bis zur Invasionsreife begiinstigende Naturboden, vor allem mit Grasbewuchs, kann nur in einigen Gehegen durch Sand- oder Kiesaufschüttung bzw. durch betonierten Untergrund ersetzt werden. Alle anderen Ausläufe und Freiflächen bieten infolge der feuchten Bodenverhältnisse gute Entwicklungsbedingungen für exogene Helminthenstadien und sind zudem kaum oder nur mit erheblichem personellem und finanziellem Aufwand bei Anwendung hierfür geeigneter Methoden (Enigk u. Eckert 1960, Encke 1966, Enigk u. Hilbrich 1968) zu desinfizieren. Mendheim und Stein bacher wiesen schon 1956 darauf hin, daß man in neuerer Zeit in Zoologischen Gärten, durch die Anforderungen eines Schaubetriebes bedingt, mehr und mehr zur Herdenhaltung verschiedener Großtierarten auf weiten Rasenflächen übergeht. Da in weiträumigen Gehegen die Tiere stets bestimmte Plätze bevorzugen, ist häufig die Ent- 


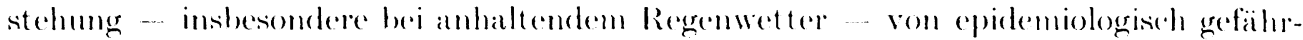
lichen morastigen Stellen, die manchen Tieriuten als Suhle dienen, nieht zu vermeiden. Aus diesen Grïnden kamn also anch eine intensiv durehgefïhrte Prophylaxe alleine nicht ausreichen. pflanzenfressende Huftiere vor endoparasitäen Helminthen zor sehiitzen. Hierzu gehören stets laufende koproskopische Untersuchungen und zum richtigen \%eitpunkt die Therapie mit geeigneten Medikamenten.

In den vergangenen Jahren beschäftigte uns besonders die jahreszeitliche Verschiedenheit der Ausscheidungsraten von Wurmeiern und -larven durch Zootiere, um bei rechtzeitiger Behandlung einer starken Gehegeverseuchung mit Reinvasion der Tierbestände und größeren Jingtierverlusten infolge Erstinfektionen zuvorzukommen. Mehrere Arbeiten (Schätzle 1964, Neunhoeffer 1965, Krumbacher 1965) wiesen eine deutliche Saisondynamik, wie sie auch von Nematoden bei Haustieren bekannt ist, nach. Daß hier neben den Eigenheiten der verschiedenen Wurmfamilien. Art und Haltung der Wirtstiere sowie klimatische Eaktoren von Einfluß waren, ist als selbstverständlich anzusehen. Für die Wiederkäıer lag der Höhepunkt der Ausscheidung von Eiern der Trichostrongyliden und Strongyliden mit tierartlichen und individuellen Abweichungen in den Monaten April bis August; eine Zunahme wurde jedoch verschiedentlich schon in Februar und März beobachtet. Der geringste Eiausstol erfolgte von November bis Januar. Die Metastrongyliden ließen in der Menge ihrer ausgeschiedenen Larven keine saisonalen Unterschiede erkennen. Auch Trichuris-Eier waren das ganze Jahr über in verschiedener. meist jedoch geringer Anzahl im Kot enthalten. Die Equiden schieden in einem Tierpark die meisten Eier von großen und kleinen Strongyliden in den Monaten Juli bis September aus, während anderenorts die Höhepunkte schon im Mär\% und April auftraten. Eier von Parascaris equorum erschienen in besonders großer Kahl von März bis Juli, bei einigen Tieren aber bereits im Februar (Forstner 19ti7). Wie die an unserem Institut durchgeführten Arbeiten von Krïger (1966) und Kaster (1974) ergaben, sind nur wenige Rundwurmarten der \%ootiere aus den Ursprungsländern eingeschleppt. Bei einigen der Wiederkäuer konnten Vertreter der Gattungen Camelostrongylus und Marshallagia, bei den Equiden verschiedentlich Gyalocephalus und bei Zebras spezifische Trichonema-Arten sowie rermutlich Strongylus asini festgestellt werden. Alle untersuchten \%ootiere beherhergten hauptsächlich Nematoden. die auch bei unseren Haustieren schmarotzen. Die Ursache hierfiir liegt in dem ständigen Kontakt mit der örtichen Fauma (Rosicky et al. 1967).

Medikamente für die Entwurnung von \%oo- und Wildtieren miissen ganz bestimmte Voraussetzungen erfiillen. Da stets der gesamte Bestand zu behandeln ist - die Therapie des Einzeltieres stellt eine mitunter notwendige Ausnahme dar - , hat ein Wurmmittel als erstes die Anforderung weitgehender Ungiftigkeit zu erfïllen. Gewöhnlich dem Kraftfutter beigemischt, darf es weder bei jungen, geschwächten noch graviden Tieren zu Unverträglichkeiten führen. Dosierungsschwierigkeiten beginnen schon beim Schätzgewicht der zu behandelnden Tiere und treten besonders bei Herdenverabreichung infolge der individuell verschiedenen Mengenaufnahme des Kraftfutters und damit des Medikaments in den Vordergrund. Ferner diirfen Geruch und Geschmack auch empfindliche Makrosmaten nicht von der vollen Annahme abhalten. Nicht zuletzt muß die Stabilität des Wirkstoffes in der Futtermischung gewährleistet sein. - Neben der erwähnten hohen toxischen Toleranz sollte ein solches Präparat auch in unterschwelligen Dosen, zumindest bei wiederholter Verabreichung, einen vermiziden Teileffekt besitzen und bei voller Dosierung möglichst viele Wurmarten erfassen. Weiterhin ist es im Tier- 
park cin betriebstechnischer Vorteil, wenn Verträglichkeit und Wurmwirksamkeit seinen Einsat\% bei verschiedenen Tiergruppen (\%. B. Equiden. Ruminantier und Suiden) gestatten.

Die pharmazeutische Industrie entwickelte in den vergangenen Jahren mehrere ..Breitspektrumanthelminthika“, die in ihren Eigenschaften den aufgereigten Erfordernissen nahekommen. Vorausgeschickt sei jedoch, daß keines dieser Vermizide das wohl kaum erreichbare Ideal gleichguter W'irkung auf T'rematoden, Cestoden und Nematoden erfiillt. Selbst spezifische Rundwurmmittel sind nicht gegen alle vorkommenden endoparasitische Nematoden wirksam und schwer bekämpfbare Arten bediirfen höherer Dosierung. Auch gibt es bis heute kein Anthelminthikum, das larvale Stadien während ihrer (iewebsphase in vollem Unfang schädigt (I) unc:an 1974). Wohl aber erfassen die modernen Präparate praeadulte Darmformen, und manche von ihnen nehmen den mit dem Kot abgehenden Eiern einiger Wurmarten die Fähigkeit zur Larvenbildung.

Vielversprechende Anthelminthika sind die seit Einfiihrung des Thiabendazols durch Brown und Mitarbeiter im Jahre 1961 bekannten Benzimidazolabkömmlinge. Von den zahlreichen Weiterentwicklungen in jünster Zeit sind mehrere noch im Versuchsstadium oder derzeit in der Bundesrepublik Deutschland noch nicht erhältlich. Wir verwendeten fiir unsere Untersuchungen Mebendazol, ein Präparat das von der Janssen Pharma(entica in Beerse/Belgien hergestellt wird und bei uns als Telmin ${ }^{1}$ und Mebenvet ${ }^{1}$ auf den Markt gelangte.

Mebendazol, chemisch ein Methyl 5(6)-benzoyl-2-benzimidazolcarbamat, ist ein weißes bis gelbliches Pulver, chemisch stabil, von extrem geringer Löslichkeit $(<0,01 \%$ in Wasser) und mit einem S(hmelzpunkt bei $280^{\circ} \mathrm{C}$. Seine vermizide Wirkung beruht, wie an verschiedenen Rund- und Bandwurmarten festgestellt wurde, auf einer Hemmung der Glukoseaufnahme durch die Helminthen und wahrscheinlich auch einer Blockierung des Glukosetransports (van den Bossche 1972; van den Bossche u. Nollin 1973). Wie mehrere von der Herstellerfirma veranlaßte Untersuchungen an einem unfangreichen Tiermaterial ergaben, vertragen Schafe, Ziegen und Kälber selbst die 40fache Dosis bei einmaliger Verabreichung, ohne klinische Erscheinungen zu zeigen, und auch bei 4 facher Überdosierung pro Applikation an ja aufeinanderfolgenden Tagen traten weder Veränderungen bei der klinischen, noch bei der patho- und histopathologischen Entersurhung auf. Auch die Blut- und Serumwerte blieben in normalen Grenzen. Zu ähnlich guten Ergebnissen gelangte Marsboom (1973), der Ponys sogar bis zu 74 Tagen täglich mit der mehrfachen Normaldosis oral behandelte. Auch Bennett et al. (1974) sahen keine negativen Auswirkungen bei Versuchen mit insgesamt 38 Pferden, die einmal die $40 \mathrm{fache}$, oder auf 15 Tage verteilt, die $90 \mathrm{fache}$ Menge der therapeutischen Dosis erhielten. Unfangreiche Prïfungen an mehreren Tierarten vor und während der Trächtigkeit sowie die Untersuchung der Foeten bzw. der Jungtiere ergaben, daß Mebendazol die Fertilität nicht beeinträchtigt und keine embryotoxischen (Ausnahmen bildeten in wenigen Fällen mit hohen Dosen behandelte Ratten) oder teratogenen Eigenschaften besitzt. - Infolge seiner Geschmack- und Geruchlosigkeit wird das Mittel, im Futter eingemischt verabreicht, gewöhnlich ohne Zögern aufgenommen. Die Ausscheidung erfolgt zum größten Teil unverändert. Absorbiertes Mebendazol verläßt den Tierkörper nach Abbau in der Leber innerhalb von 24 Stunden mit Harn und Kot. Nach 14 Tagen ist das Fleisch behandelter Tiere frei von Rückständen.

\footnotetext{
1 Janssen GmbH, Vet. med. Abteilung, Düsseldorf.
} 
Die anthelminthische Wirkung von Mebendazol erst reckt sich bei Hauswiederkäuern nach Firmenberichten auf alle im Magendarmtrakt vorkommenden Rundwiimer (Tri(hostrongyliden, Strongyliden, Capillarien und Trichuris). Diese Angaben werden von Janisch und Varga (197t) bestätigt. Auch die in der Lunge schmarotzenden Dictyocaulus-Arten und Protostrongyliden sollen erfaßt werden. Intraruminale Verabreichung von $10 \mathrm{mg} / \mathrm{kg} \mathrm{KGW}$ an Schafe und $15 \mathrm{mg} / \mathrm{kg} \mathrm{KGW}$ an Kälber beseitigten Moniezia-Befall vollständig. Eier von Haemonchus contortus hatten 20 Stunden nach der Behandlung von Schafen mit $15 \mathrm{mg} / \mathrm{kg} \mathrm{KGW}$ ihre Entwicklungsfähigkeit verloren. Guilhon et al. (1972a) berichten aus Frankreich, daß die üblichen Magen-Darmnematoden der Schafe durch einmalige orale Gaben ( 8 bis $35 \mathrm{mg} / \mathrm{kg} \mathrm{KGW}$ ) gut zu beeinflussen sind. Dur(h Sektion, eine Woche nach Mebendazolverabreichung an Lämmer in Dosen von $20 \mathrm{mg} /$ kg KGW, stellten Kates und Mitarbeiter (1974) die noch vorhandene Wurm- bzw. Larvenzahl fest. Im Vergleich mit den unbehandelten Kontrollen ermittelten sie eine gute Wirkung gegen Oesophagostomum $(100 \%)$, Ostertagia $(99 \%)$, Nematodirus $(96 \%)$ und Trichuris $\left(91^{\circ}{ }_{0}\right)$, eine weniger zufriedenstellende gegen Trichostrongylus $\left(81_{0}^{\circ}\right)$, Haemonchus $\left(53{ }_{0}\right)$, Strongyloides $\left(43^{\circ}\right)$ ) und Moniezia $\left(41^{\%}\right)$. Das schlechte Ergebnis bei Haemonchus sehen sie in der Verwendung von Stämmen begrïndet, welche gegeniiber anderen Benzimidazolpräparaten bereits resistent geworden waren. Auch 4. und 5. Larvenstadien der häufigsten Magen-Darmstrongyliden wurden zu einem beachtlichen Prozentsatz erfaßt. - Untersuchungen an Wildwiederkäuern (Reh-, Rot-, Dam-, Sika-, Muffelund Gamswild) von Kutzer und Mitarbeitern (1974) erbrachten einen Therapieerfolg von rund $95^{\circ}$, bei Trichostrongylidenbefall. Ferner wurden Strongyliden, Ancylostomatiden, Trichuriden und kleine Lungenwïmer beeinflußt. Das Wild erhielt Mebendazol in geschrotetem Kraftfutter 10 Tage lang täglich in Dosen von $3 \mathrm{mg} / \mathrm{kg} \mathrm{KGW}$ oder 3 Tage täglich $15 \mathrm{mg} / \mathrm{kg} \mathrm{KGW}$. Beide Dosierungen hatten erhebliche Einwirkungen auf die kleinen Lungenwürmer. Wie obige Autoren im Exaktversuch an Schafen feststellen konnten, nahm die Zahl der ausgeschiedenen Muellerius capillaris-Larven vom 8 . bis gegen den 25. Tag nach Behandlungsheginn deutlich ab und sistierte zeitweilig ganz. Peitschenwïrmer (Trichuris) beim Elchwild wurden durch $10 \times 3 \mathrm{mg} / \mathrm{kg} \mathrm{KGW}$ Mebendazol nur bei einem von 3 Tieren abgetrieben (Meier 1974), und auch Dollinger (1973) berichtet nur von einem Teilerfolg bei einem im Tierpark gehaltenen Elch (5) $\mathrm{mg} / \mathrm{kg}$ und $4 \times 3 \mathrm{mg} / \mathrm{kg} \mathrm{KGW})$.

Zahlreiche Untersuchungen an Equiden verschiedener länder belegen den ausgezeichneten vermiziden Effekt von Mebendazol gegen die häufigsten Rundwurmparasiten dieser Tiere. So konnte durch Kotuntersuchung auf Wurmeier und Zählung der abgegangenen Würmer in mehreren firmeneigenen und auf Vertragshasis durchgefïhrten Arbeiten an erwachsenen Pferden und Fohlen eine 96-100\%, ige Wirkung auf Oxyuren, Trichonemen, Strongylus-Arten und Paraskariden nachgewiesen werden. Die Dosierung betrug für Fohlen 2 und für ausgewachsene Tiere $4 \mathrm{~g}$, das entsprach $8-10 \mathrm{mg} /$ $\mathrm{kg}$ KGW. Auch die einmalige Verabreichung von nur $0,75 \mathrm{~g}$ je Pferd führte zu einer $100^{\circ}$ igen Reduktion der Eier von Strongylus spec. ab dem 3. Tag. Saupe und Nitz (1972) erhielten ähnliche Ergebnisse nach Behandlung von 68 Pferden mit je 4 g. Ei- und Larvenzählungen nach Anzucht in der Kultur über einen Zeitraum von 4 Wochen zeigten im Durchschnitt eine Abnahme der Wurmeiausscheider um 98,3 bis 99,4\%. Die am Befall beteiligten Wurmarten waren Parascaris equorum, Strongylus vulgaris, S. edentatus, S. equinus und kleine Pferdestrongyliden. In jüngster Zeit berichtete auch Sommer (1975) von guten Erfolgen bei 56 Warmblïtern nach einmaliger Zufütterung von $4 \mathrm{~g}$ 
pro Tier. Ehenso stellten Walker und Knight (1972) nach Eingabe von Einzeldosen zwischen $1 \mathrm{u}$ und $13 \mathrm{mg} / \mathrm{kg} \mathrm{KGW}$ an Pferde und Ponys verschiedener Rassen durch quantitat ive dreimalige Nachuntersuchung eine Reduktion der Strongylideneier 1 m praktisch 10(1)" fest. 42-45 Tage p.a. traten erstmals wieder Eier im Kot auf. Mittels Kotkulturen und Ident ifizierung der 3. Larven konnte gezeig t werden, daß alle beteiligten Palisadenwiimer durch das Medikament gleichermaßen erfaßt wurden. Ostmann (1973) erzielte mit $8,8 \mathrm{mg} / \mathrm{kg} \mathrm{KGW}$ gegen Parascaris equorum, große und kleine Strongyliden sowie Oryuris equi eine 92-100\% ${ }^{\circ}$ ige Wirkung, während bei sonst vergleichbaren Ergebnissen Neave und Callear (1973) durch Gaben von $2, \overline{\mathrm{mg}} / \mathrm{kg} \mathrm{KGW}$ an 3 aufeinanderfolgenden Tagen eine Reduktion der Eizahl um 99,30", erreichten. Nach Einzeldosen zwischen 10 und $20 \mathrm{mg} / \mathrm{kg} \mathrm{KGW}$ war der Kot innerhalb von 2-4 Tagen frei von Strongylideneiern, welche dann nach 4-6 Wochen wieder erschienen. Daraus schließen Guilhon et al. (1972 b), daß Wanderlarven nicht abgetötet werden oder eierlegende Wurm-qo in dieser Zeit ihre Aktivität wiedererlangen. Bei Zooequiden war die Behandlung mit $6 \mathrm{mg} /$ kg K( $\mathrm{xW}$ gegen Spulwiirmer und Strongyliden hoch wirksam (Dollinger 1973). Mehrere kritische Tests, bei denen nach Medikamentverabreichung die Anzahl der abgetriebenen, im Kot feststellbaren Würmer und ihrer Larvenstadien mit der Anzahl der später bei Sektion des Wirtstieres aufgefundenen Exemplare verglichen werden, wiesen Mebendazol als ausgezeichnetes Mittel gegen die häufigsten Wurmparasiten der Pferde, Ponys und Esel aus (Reinecke u. Le Roux 1972, Bennett 1973, Bradley u. Radhakrishnan 1973). Einmalige Gaben von $\overline{0}-10 \mathrm{mg} / \mathrm{kg} \mathrm{KGW}$ zeigten höchste Wirkung auf adulte große und kleine Pferdestrongyliden, Parascaris equorum und Oxyuris equi sowie deren 4. Larven. Wie auch die Untersuchungen mit Eizählung erkennen ließen, sind Oxyuren wohl mit noch geringeren Dosen sicher zu erfassen, im Hinblick auf die schwerer angreifbaren Trichonema-Arten und Strongylus edentatus sollten jedoch $5 \mathrm{mg} / \mathrm{kg} \mathrm{KGW}$ nicht unterschritten werden. A uf Habronema, Trichostrongylus und Strongyloides hatte Mebendazol keinen Einfluß. - Auch Equiden vertragen das Präparat sehr gut. Noch die 10 fache Dosis $(80 \mathrm{mg} / \mathrm{kg} \mathrm{KGW}$ ) ruft keinerlei Nebenerscheinungen hervor. $50 \mathrm{mg} /$ kg KGW iiber 2 Monate täglich verabreicht, führten weder zu klinischen noch haematologisch oder serologisch nachweisharen krankhaften Veränderungen. Die LD) 50 beim Pferd liegt iiber $400 \mathrm{mg} / \mathrm{kg} \mathrm{KGW}$.

Uher gute Erfolge mit Mebendazol bei der Bekämpfung endoparasitärer Helminthen anderer Tierarten berichten mehrere Autoren, so, $u$ m nur einige zu nennen, beim $\mathrm{S}$ ( hwein Nitz (1974), bei Fleischfressern Grevel und Eckert (1973), Forstner (1974), bei Affen Hohenester (1973) und beim Gefliigel Enigk und Mitarbeiter (1973) sowie Wiesner (1973).

In eigenen Untersuchungen setzten wir Mebendazol in den Jahren 1972 bis 1974 im Rahmen der jährlichen Routineentwurmungen an Wiederkäuern und Equiden des Münchner Tierparks Hellabrunn ein.

Aus der Gruppe der Wiederkäuer wurden über 90 (93-99) in Herden gehaltene, nach Art getrennte Tiere durch Sammelkotproben kontrolliert. In den ersten beiden Jahren erstreckten sich die Überprüfungen auf Wapitis, Virginia- und Maultierhirsche, Gemsen, Alpensteinböcke sowie Sibirische und Nubische Steinböcke, Schraubenhornziegen, Argalis, Kreishorn- und Mähnenschafe, Mufflons, Tahre, Ture und 1972 auch auf Zwergmuntjaks. 1974 wurden Wapitis, Virginia- und Maultierhirsche, Argalis und Kreishornschafe nicht mehr in den Behandlungsplan aufgenommen, dafür Sambur- und Barasinghahirsche, Sika, Elche, Rentiere, Marale und Milus, Nilgau- und Hirschziegenanti- 
lopen, Dorkas- und Kropfgazellen sowie Springhörke. - Die Equiden konnten infolge ihrer nächtlichen Einzelaufstallung individuell untersucht und behandelt werden. 1972. und 197:3 waren es jeweils 37 Tiere und zwar (Arant-. Damara-, Berg- und (irevy-\%ebras, ferner Tarpane, Prowalski-Pferde und Halbesel. Da die Kulane (1) Tiere) im zweiten Jahr während des gesamten Überpriifungszeitraumes von (i Monaten keine Wirmeier mehr ausschieden, schlossen wir sie 1974 von weiteren Untersuchungen aus.

Insgesamt wurden 1380 Wurmeieranreicherungen mit Hilfe des Flotationsverfahrens $\left(\% \mathrm{nCl}_{2}: \mathrm{NaCl}=1: 2\right.$ als gesättigte wässerige Lösungen $)$ durchgefuihrt und 785 Kotproben im Auswanderverfahren nach Baermann-Wet \%el zum Lungenwurmlarvennachweis ausgewertet. Im ersten Untersuchungsjahr erfolgte die Differenzierung der aufgrund ihrer Morphologie nicht zu trennenden Fier der verschiedenen Pferdestrongyliden und der Magen-Darmstrongyliden der Wiederkäuer nach Anzucht der Larven III von jeder befallenen Tierart vor und nach der Behandlung.

Das Mebendazol wurde in Reinsubstanz dem Kraftfutter untergemischt und jeweils 14 Tage lang verabreicht. Die Dosierung errechneten wir iiber das geschätzte Gesamtgewicht und den Kraftfutterverbrauch; sie betrug fïr Wiederkäuer 1, 3 oder $5 \mathrm{mg} / \mathrm{kg}$ KGW und für Equiden 0,75 bzw. $1 \mathrm{mg} / \mathrm{kg} \mathrm{KGW}$.

Gehege, bei denen es die Bodenbeschaiffenheit zuließ (bei Sibirischen und Nubischen Steinböcken, Turen, Schraubenhornziegen und Mähnenschafen) wurden zu Beginn dieser Versuche im Jahre 1972 zweimal nach vorausgegangener Grobreinigung desinfiziert und zwar 1-3 Tage nach Behandlungsbeginn und unmittelbar im Anschluß an die Applikationsperiode. Hierzu diente Lysococ ${ }^{\mathrm{R}}$ der Firma Schülke und Mayr, die das Präparat dankenswerter Weise zur Verfiigung stellte.

Ein Tierpark kann seine Aufgabe nur mit Tieren in Schaukondition erfuillen. Die zur Gesunderhaltung des Bestandes not wendigen Ent wurmungen sind deshalb vorrangigpraktische tierärztliche Verrichtungen, die eine wissenschaftliche Auswertung nur in diesem Rahmen zulassen. Aus diesen Gründen ist es nicht immer möglich, unbehandelte Kontrollgruppen sinnvoll im Untersuchungsplan unterzubringen. Zumindest haben hier: Kontrollen nicht den bei wissenschaftlicher Versuchsanstellung geforderten Aussagewert. Mit ihnen soll ja der Unterschied zwischen behandelten und unbehandelten Tieren, also die Wirkung eines Medikaments, belegt werden. Das setzt aber die Cleichartigkeit aller Bedingungen, eben mit der einen Ausnahme des zu priifenden Faktors, voraus. Demnach können z. B. die Przewalski-Pferde nur mit größten Einschränkungen als Kontrollgruppe zu der behandelten /ebraherde gelten. Verschieden ist schon die Tierart mit anderer Empfänglichkeit für Parasiten, dann die Kopfzahl, Alter, Verhaltensweisen, Gehegebeschaffenheit, vieles andere mehr und nicht zuletzt Art und Grad der Verwurmung sowie der Eiausscheidung. Diese Differenzen sind bei verwandtschaftlich weit auseinanderstehenden Wiederkäuern noch erheblich größer. Vielversprechender, und die einzige Möglichkeit zur Beurteilung der Wirkung eines Wurmmittels bei Zootieren, sind wiederholte grïndliche Untersuchungen vor und nach der Behandlung.

Die Voruntersuchungen im Herbst 1972 sollten zunächst eine Aussage über die Höhe der anzuwendenden Dosierung und die Aufnahme des medikierten Futters erbringen. Nicht unwesentlich schien uns dabei, die für die jeweiligen Gehege verantwortlichen Tierpfleger an die regelmäßige und sachgerechte Probenentnahme, an eine verläßliche Beschickung der Tröge mit dem wurmmittelhaltigen Kraftfuttergemisch und die intensive Beobachtung der Tiere, insbesondere bei Herdenfütterung, zu gewöhnen. Die Ei- und Larvenausscheidung wurde dreimal in zweiwöchigen Abständen vor der Behandlung, 
die iblee 14 Tage vom 10.-23. 10. 1972. mit täglichen Dosen von $1 \mathrm{hzw} .3 \mathrm{mg} / \mathrm{kg} \mathrm{K}(\mathrm{xW}$ fiir Wiederkäuer und 0.75 mg/kg K(ill fiir Equiden vorgenommen wurde, fest gestellt. Während des Verabreichungs\% it rammes erfolgten 2 \%wischenunt ersuchumgen (am 3 . und 9. Tag nach Beginn des Mebendazolangebots) und anschließend 2 Nathuntersuchungen (am 3. und 17. Tag nach Absetzen des Medizinalfutters).

Unter den Wiederkäuern waren bei 4 von 15 Tiergruppen keine parasitischen Entwicklungsstadien nachzuweisen. 1)iese Herden (Virginia- und Maultierhirsche, Tahre und /wergmuntjaks) schjeden von den weiteren Untersuchungen ror der Medikation aus. Bei den befallenen 11 Wiederkäuergruppen konnte zu dieser. Jahreszeit generell nur eine geringe Anzahl von Wurmeiern und Lungenwurmlarven festgestellt werden. In jeder Herde befanden sich Ausscheider von Magen-Darmstrongylideneiern und in 9 solche von Larven kleiner Lungenwürmer (nicht bei Wapitis und Nähnenschafen). Verschiedentlich waren ('apillaria (ror allem bei Kreishornschafen und Schraubenziegen), Trichuris (vermehrt bei Kreishornschafen) und Strongyloides (stärkere Ausscheidung ebenfalls bei Kreishornschafen) nachweisbar. Die Magen-Darmstrongylideneier konnten aufgrund der Larvenzucht den Gattungen Ostertagia, Trichostrongylus, Haemonchus sowie ('habertia-Oesophagostomum, die Lungenwurmlarven C'ystocaulus, Protostrongylus, Muellerius und Neostrongylus zugeordnet werden. - Die zweimalige Kontrolle während des Behandlungszeitraumes ergab, zumindest bei der zweiten Überprifung, einen leichten Riickgang der Eizahlen. I)ie später erfolgten Nachuntersuchungen zeigten eine deutliche Verminderung der Magen-Darmstrongylideneier, die sich in der Anzahl festgestellter Eier pro Herde. wie auch in der Zahl der ausscheidenden Tiergruppen erkennen ließ. Die prozentuale Zusammensetzung der am Magen-])armstrongylidenbefall beteiligten Wurmgenera hatte sich, wie nach erneuter Larvenzucht und -differenzierung festzustellen war, kaum geändert. Trichuris. Capillaria und Strongyloides wurden unterschiedlich beeinflußt. Ein eindeutiges Sistieren der Ausscheidung dieser Würmer war nicht gegeben. Der therapeutische Effekt auf kleine Lungenwïrmer blieb aus. - Ein Vergleich mit den zur Kontrolle mbehandelt gebliebenen Mähnenschafen und Argalis ergab jedoch, daßs auch die Ausscheidung von Magen-Darmmematodeneiern bei diesen Tieren während des Versuchszeit raumes weitgehend zuriickging. Dies wurde besonders bei den Mähnenschafen beobachtet, deren Gehege desinfiziert worden war. Möglicherweise liegt in der geringen Reinfektionsmöglichkeit, eventuell im /usammenhang mit der fortgeschrittenen Jahreszeit der (rund fiir das Aufhören der Wiausscheidung.

Bei kritischer Betrachtung dieser Ergebnisse muls gesagt werden, daß bei allen befallenen Tieren erfahrungsgemäß die Anzahl der im Kot erscheinenden Wurmeier auf den Winter zu absinkt und demnach eine anthelminthische Wirkung durch die \%ufüterung des Medikaments in nachweisbarem Unfang nicht gegeben war, zumal keinerlei Unterschiede zwischen den (iruppen verschiedener Dosierung ( $1 \mathrm{mg} / \mathrm{kg}$ und $3 \mathrm{mg} / \mathrm{kg}$ $\mathrm{K}(\mathrm{iW})$ auftraten. Die Ursache hierfiir ist jedoch nicht nur in der zu gering veranschlagten Wurmmittelmenge zu sehen, sondern hauptsächlich in dem zu hoch eingeschätzten täglichen Kraftfutterbedarf der /oowiederkäuer. Ganz besonders störend wirkte sich die zur \%eit des Mebendazolangebots eingetretene Brunft aus. Dadurch nahmen viele Tiere die Medikation nur sporadisch oder iiberhaupt nicht an. Der Mißerfolg dieses ersten Versuchsdurchganges lag also an der hochgradigen Unterdosierung des Mebendazols und nicht an seinen Wigenschaften.

Die gleichzeitig an Equiden vorgenommenen Überprïfungen ergaben einen Wurmbefall bei 30 Tieren $\left(=81^{\circ}, 0\right)$. I)avon schieden 27 Eier von Strongyliden und 8 von Paras- 
caris aus. Bei 1:3 von 27 Tieren fanden sich regelmäßig Strongylideneier, wenn anch meist mur in geringer Anzahl. im Kot. Durch Larvenzucht konnten sie den Arten Strongylus vulgaris. s. equinus. S. edentatus und, zahlenmäBig am häufigsten, den kleinen Palisadenwiumern zugeordnet werden. Spulwumeier erschienen bei $t$ von 8 befallenen Equiden regelmäßig. - Durch ein nicht von uns verschuldetes Versehen wurde dem Kraft futter fiir die Pferdeartigen Mebendazol in zu geringer Menge eingemischt. Durch Verdoppelung des Angebots (Adulte $2 \mathrm{~kg}$, Fohlen $1 \mathrm{~kg}$ ), die verabreichte Menge nahmen alle. Tiere vollständig auf, konnte die Dosierung immerhin noch auf täglich $0,7 \overline{m g} / \mathrm{kg}$ KGW angehoben werden. Schon die \%wischenuntersuchungen während des 14-tägigen Applikationszeitraums (am 3. und 9. Tag) zeigten eine deutliche Abnahme der ausigeschiedenen Strongyliden- und Parascaris-Eier. Letztere waren bei der zweiten Überpriifung bereits nicht mehr nachweisbar. Während 2 Tage nach Abschluß der Behandlung nur noch 2 Tarpane vereinzelt Strongylideneier im Kot aufwiesen. war dieser bei allen Tieren nach weiteren 16 Tagen völlig wurmeierfrei. - Als unbehandelte Kontrollgruppe diente die Przewalski-Herde, bestehend aus 8 Tieren, bei denen ïber den gesamten Untersuchungszeitraum geringgradig Strongylideneier festgestellt werden konnten. Die später analog vorgenommene Entwurmung führte zu demselben Ergebnis. Am 3. und 10. Tag nach Absetzen des medikierten Futters waren keine Wurmeier mehr festzustellen. Bei einer letzten Nachuntersuchung am 23. Tag traten wieder vereinzelt Strongylideneier auf. Wie die Bestimmung der angezïchteten larven ergab, handelte es sich hierbei um Geschlechtsprodukte von Trichonemen und Strongylus edentatus.

Mebendazol führte selbst in der sehr niederen Dosierung von $0,75 \mathrm{mg} / \mathrm{kg} \mathrm{KGW}$ bei 14tägiger Verabreichung an Equiden zum Sistieren der Eiausscheidung von Spul- und Palisadenwïmern. Die Aufnahme des medikierten Kraftfutters war stets vollständig. Da die Tiere einzeln gefüttert wurden und die Kotproben von Einzeltieren stammiten. sind diese Ergebnisse gesichert. Das spätere Wiedererscheinen von Trichonema- und Strongylus edentatus-Eiern ist dem geringen Mebendazolangebot zuzuschreiben. Wie Bradley und Radhakrishnan (1973) zeigten, sind diese Wurmarten gegeniiber Mebendazol etwas weniger empfindlich.

Bei den Hauptuntersuchungen im Jahr 1973 begannen wir mit der koproskopischen Überpriifung der Tierbestände schon im Februar, $1 m$ die Behandlung rechtzeitig heim Frïhjahrsanstieg der Eiausscheidung durchführen zu können. Fs stellte sich jedoch heraus, da 3 die anfänglich bei den jeweiligen Herden bzw. Tieren nur in geringer Zahl nachweisharen Wurmeier auch in späteren Monaten nicht wesentlich zunahmen. So wurden die Wiederkäuer und Equiden bis zur zweiten Hälfte des Monats Juli 9mal in etwa 14tägigen Abständen kontrolliert. Im Hinblick auf die Gefährdung der Jungtiere, die unter Helminthosen erfahrungsgemäß erheblich zu leiden haben, erhielten dann die Wiederkäuer, deren tatsächlicher Kraftfutterverbrauch 2 Wochen lang zuvor für jede Herde genau festgestellt wurde, iiber 14 Tage (24.7.- (i. 8. 1973) täglich 5 $\mathrm{mg} / \mathrm{kg} \mathrm{KGW}$ (620 ppm) Mebendazol. Die Ửberprüfung des Behandlungserfolges geschah durch 2 Nachuntersuchungen. wovon die erste noch während des Behandlungszeitraumes am 10. Tag nach Applikationsbeginn und die zweite eine Woche nach Abschluß der Mebendazolverabreichung erfolgte. - Die Equiden bekamen vom 17. - 30. 7.1973 pro Tag $1 \mathrm{mg} /$ kg KGW (400 ppm) des Wurmmittels ebenfalls im Kraftfutter; die Nachuntersuchungen wurden am 3. und 17. Tag p.a. durchgefiihrt.

Von den 14 in die Überprüfungen einbezogenen Wiederkäuerherden (insgesamt 93 Tiere) schieden alle, mit Ausnahme der Virginiahirsche. Eier von Magen-Darmwïrmern 
aus. Bei 12 Gruppen (nicht Virginia- und Maultierhirsche) lag gleichzeitig ein Befall mit kleinen Lungenwiirmem vor. Magen-I)armstrongylideneier fanden wir stets im Kot der Waplitis und fast immer bei Mufflons und Kreishornschafen, während die restlichen Tiergruppen bei den 9 Voruntersuchungen bis zu tmal als Ausscheider ermittelt wurden. Capillaria-Befall war häufig bei Kreishornschafen, mehrere Male bei Mufflons, Steinböcken, Argalis und mitunter bei Gemsen. Turen. Tahr. Schraubenziegen und Maultierhirschen nachzuweisen. Bei diesen Herden, nicht jedoch bei Steinböcken und Mufflons, stellten wir verschiedentlich auch Trichuris-Eier fest. Kreishornschafeschieden bei o Voruntersuchungen Eier von Strongyloides aus, Gemsen und Steinböcke zweimal, Wapitis, wie auch die Argalis, nur einmal. In 3 Herden konnten jeweils bei nur einer der Ưberprïfungen Eier von Nematodirus, bei den Alpensteinböcken dreimal von Moniezia diagnostiziert werden. Lungenwurmlarven der Gattungen Muellerius, Cystocaulus, Proto- und Neostrongylus, in der Reihenfolge ihrer Häufigkeit genannt, traten bei Schraubenhornziegen, Nubischen Steinböcken und bei Kreishornschafen meist in größerer Zahl, bei Turen, Alpensteinböcken und Argalis in ïber der Hälfte aller Kotkontrollen auf, seltener bei den restlichen Herden.

Am 10. Tag der Mebendazolverabreichung, wie auch eine Woche nach Behandlungsende, waren die Proben aller Tiere frei von Wurmeiern jeglicher Art. Protostrongylidenlarven konnten nur bei Tahren und Mähnenschafen vereinzelt bei der ersten, in keinem Fall aber bei der zweiten Untersuchung nach Wurmmittelangebot aufgefunden werden.

Mebendazol in einer Dosierung von $5 \mathrm{mg} / \mathrm{kg} \mathrm{KGW}$ täglich iiber 14 Tage mit Kraftfutter verabreicht ist also in der Lage, die $\mathrm{Ei}$ - und Larvenausscheidung der häufigsten endoparasitischen Wïrmer bei den hier untersuchten Arten von Zoowiederkäuern völlig zım Erliegen zu bringen. Besonders zu erwähnen ist die gute Wirkung auf die bisher schwer erfaßbaren kleinen Lungenwïmer, wie auch auf Trichuris und Capillaria.

Unter den Equiden (37 Tiere) schieden die Kulane ( 6 Tiere) während der Überpriifungen im .Jahr 1973 niemals Wurmeier aus. Von den restlichen 31 Tieren beherbergten 27 Darmhelminthen, und zwar 22 von Strongyliden, die interessanterweise bei Bergzebras (5 Tiere) nicht nachweisbar waren, und 10 Parascaris, welche nur bei den Zebras vorkamen, nicht dagegen bei Przewalski-Pferden, Tarpanen oder Kulanen. 15 der 22 mit Palisadenwïrmern befallenen Equiden hatten viermal oder öfter, davon 7 stets (bei 9 Voruntersuchungen) Strongylideneier im Kot. Die Proben enthielten vereinzelt bis mittelgradig, durchschnittlich geringgradig, diinnschaìige Nematodeneier. Die Hälfte der Parascaris-Träger, also §’ Tiere, schieden regelmäßig, 8 viermal oder häufiger, im Schnitt mittelgradig Spulwurmeier aus. - Die Nachuntersuchungen am 3. und 17. Tag nach Beendigung der Wurmmittelverabreichung ergaben, da $\beta$ die Proben aller in die Ưherprüfung eingeschlossenen Pferdeartigen frei von Wurmeiern waren.

Bei Equiden kann demnach durch 14 tägige Zufütterung von täglich $1 \mathrm{mg} / \mathrm{kg} \mathrm{KGW}$ Mebendazol die Eiablage von Pferdestrongyliden und -spulwiirmern vollständig unterbunden werden.

1974 erhoben wir den Parasitenstatus durch 6 Voruntersuchungen von anfangs März bis Ende Mai. Die 14tägige Therapie, wieder mit täglichen Dosen von $5 \mathrm{mg} / \mathrm{kg} \mathrm{KGW}$ (620 ppm) für Wiederkäuer und $1 \mathrm{mg} / \mathrm{kg} \mathrm{KGW} \mathrm{(400} \mathrm{ppm)} \mathrm{für} \mathrm{Equiden,} \mathrm{wurde} \mathrm{im} \mathrm{Juni}$ (4.-17. 6. 1974) vorgenommen. Durch dreimalige Kotüberpriifung am 3., 10. und 31. Tag nach Absetzen des Medizinalfutters stellten wir das Behandlungsergebnis fest. Als Kontrolle dienten Alpensteinböcke und Przewalski-Pferde, welche erst im Anschluß an die zweite Nachuntersuchung der anderen Tiere ebenso wie diese therapiert (vom 2.-16. 7 . 
1974) und in analogen \%eit intervallen koproskopisch weiterverfolgt wurden. - An Wiederkäuern iiberpriften wir et wa 100 Tiere aus 22 Herden. Wie hereits orwähnt, wurden einige Tiergruppen des Vorjahrs nicht mehr untersucht, dafiir jedoch andere Cerviden-, Antilopen- und (azellenarten. - Die Uberprïung der Equiden erfolgte an 25 Tieren und zwar an 10 \%ebras, 8 Tarpanen und 7 Przewalski-Pferden. 1)a die Kulane 1973 nie Wurmeier ausschieden, verzichteten wir auf ihre weitere Kontrolle.

Wurmbefallene Tiere waren in 19 Wiederkäuerherden festzustellen, nicht jedoch bei Tahren, Samburhirschen und Milus. 18 'Tiergruppen schieden Eier von Magen-Darmhelminthen und 5 Larven von Protostrongyliden (Cystocaulus. Protostrongylus) aus. Eier von Magen-Darmstrongyliden, meist nur in geringer /ahl, fanden wir im Kot von 14 Herden. Regelmäßig, d.h. bei jeder der 6 Voruntersuchungen, gelang ihr Nachweis nur bei den Hirschziegenantilopen, ‘mal bei Gemsen und Rentieren. tmal bei Nilgaus. Dorkasund Kropfgazellen sowie Mufflons, während die restlichen Wiederkäuergruppen $3 \mathrm{mal}$ oder seltener einen Befall mit diesen Nematoden erkennen ließen. Nematodirus - und Strongyloides-Eier kamen nur sporadisch hei Gemsen, Mufflons und Kropfgazellen vor (jeweils nur bei einer Untersuchung). Capillarien traten bei den Elchen häufig (5mal) auf. Sie ließen sich ferner bei Gemsen (3mal) und Sibirischen Steinböcken (2mal) öfter nachweisen und waren auch in 6 weiteren Wiederkäuerherden vorhanden. Eier von Peitschenwümern fanden wir meist in größerer Kahl und fast bei jeder Untersuchung (5mal) des Elchwildes, hin und wieder hei Kropfgazellen, selten in den Beständen der Nilgaus. Markhors und Oryxantilopen. Mähnenschafe hatten einmal Moniezia-Eier im Kot. Einzelne Larven kleiner Lungenwïrmer ließen sich bei Alpensteinbö(ken an 5, bei Dorkasgazellen in mehreren Exemplaren an 4 Überpriifungstagen, ferner auch bei Nubischen Steinböcken (2mal), Elchen und Turen (je 1mal) feststellen. - 3 Tage nach der Behandlung war der Kot aller Wiederkäuer frei von Parasitenstadien mit Ausnahme der Dorkasgazellen, bei denen jede Nachuntersuchung Cystocaulus-Larven ergab und den Elchen, welche weiterhin Trichuris-Eier ausschieden. 10 Tage p.a. traten bei Rentieren wieder vereinzelt Eier von Magen-I)armstrongyliden auf und nach einem Monat zusätzlich bei Springböcken. Dorkas, Samburhirschen und Mufflons, vermehrt bei den Hirschziegenantilopen.

Der unbefriedigende Entwurmungserfolg bei den Dorkasgazellen und Elchen ist vermutlich weniger dem Anthelminthikum anzulasten, als vielmehr den Freßgewohnheiten dieser Tiere. So nehmen z. B. die I)orkasgazellen bevorzugt die dem Kraftfutter untergemsschten Äptel aut. Bet Herdentutterung mub terner stets damit gerechnet werden, daß eines der Tiere das Wurmmittel in zu geringer Menge oder nicht regelmäßig erhält. Dieser Ausscheider, nicht selten ein stärker befallenes Jungtier, wird dann mit der Sammelkotprobe erfaßt und verfälscht das Ergebnis. In solchen Fällen fiihrt mitunter die Einzelbehandlung \% $\%$ Ziel, wie das Scheel (19157) an einem Elchkalb mit starkem Ausstoß von Trichuris-Kiern zeigen konnte. Widerspriichlich sind die Angaben iiber die Mebendazolwirkung auf Trichuris. K a tes und Mitarbeiter (1974) stellten bei Schaflämmern eine $100^{\circ}$ "ige Beseitigung derPeitschenwiirmer fest, während in unseren Untersuchungen wie auch in denen anderer Autoren (Dollinger 1973; Meier 197t) bei Elchen kein einwandfreies Ergebnis zu erhalten war. Ob es sich hierbei tatsächlich um tierartliche Unterschiede oder Unterdosierung infolge der angegebenen Grïnde handelt, ist derzeit nicht zu entscheiden. Daß gerade Peitschenwïrmer bei in Gehegen gehaltenen Wildwiederkäuern schwere Gesundheitsschäden hervorrufen können, wurde verschiedentlich beobachtet (Brglez u. Senk 1969). Das erneute Auftreten von Magen-Darmstrongyli- 
deneiem am 31. Tagr par. in einigen Herden ist. seltst wemn Mebendazol die Larvenentwicklung \% verhindern rermag. eine Folge der Reinfektion mit friber ansgeschiedenen und inzwischen ansterkmugse gewordenen Entwicklungsstadien, denn fiir einge der in dem Sammelhegriff ..Magen-1)armstrongyliden" zusammengefaßsten Wurmgattungen heträgt die Präpatent zeit weniger als 30 Tage. Ferner können die während ihrer hist ot ropen Phase durch ein Wurmmittel sehwer beeinflußbaren Larven, z. B. von Osterlagia-Arten. bis zom \%eitpunkt der letzten Nachuntersuchung längst Geschlechtsreife erreicht haben. Auch \%wischenzeitlich stattgefundene Neuinfektionen sind nicht auszuschließen. So muß das Auftreten von Nematodirus-Eiem bei Hirschziegenantilopen darauf zuriickgefiihrt werden, da in 8 Utherpriifungen von Mär\% bis .Juni bei dieser Herde niemals ein Befall mit dieser Wurmgattung festzustellen war. Die Möglichkeiten der Einschleppung sind vielfältig, jedoch hier nicht Gegenstand näherer Betrachtung. - So beurteilt, ist der Behandlungseffekt bei den Wiederkäuern mit Mebendazol durchaus den guten Erfolgen des Vorjahres vergleichbar. zumal 19-33 die ,.kritischen Tierarten" nicht. untersucht bzw. hehandelt worden sind.

Die 1974 parallel zu den Wiederkäuern an Pferdeartigen rorgenommenen Untersuchungen ergaben bei 19 von 25 Tieren einen Befall mit Strongyliden und bei i zusätzlich mit Spulwïrmern. Vor der Mebendazolzufuitterung schieden 8 der Equiden stets Eier von Palisadenwiirmern aus, $1+$ Tiere 3 mal oder öfter. Während im Vorjahr Parascaris nur bei \%ebras festgestellt werden konnte. beherbergten jetzt auch ein Tarpan und ein Przewalski-Pferd diese Parasiten. Dagegen waren 3 Bergzebras (Alter 2, 2 und 26 Jahre). seinerzeit regelmäßige Ausscheider von Spulwurmeiern, nicht mehr als solche erfaßbar. :3 der Spulwurmträger hatten bei 3 und mehr Ủberprïfungen Eier im Kot, einer davon stets. I)ie Eizahl pro Untersuchung variierte von vereinzelt bis mittelgradig. - Am 3., 10. und 31. Tag nach Absetzen des medikierten Futters waren die Proben aller Tiere, auch die der später behandelten Kontrollen, frei von Wurmeiern.

Durch die 14 tägige Zufiitterung von Mebendazol in der geringen Dosis von täglich$1 \mathrm{mg} / \mathrm{kg} \mathrm{K}$ ( $\mathrm{aW}$ gelang es auch in diesem. Jahr, bei allen 25) in die Behandlung eingeschlossenen Pferdeartigen. die Eiausscheidung von Spulwiirmern sowie großen und kleinen Sitrong.liden vollständig zu unterdriicken. Bei keiner der 3 Nachkontrollen, die innerhalb cines Monats p.a. (d.h. bis \% 4i) Tagen nach Behandlungsbeginn) durchgefüht wurden, war auch nur ein Ei der genannten Nematoden fest zustellen. ()b nun dieses Ergebnis als Folge der abtreibenden Wirkung nur auf i) armstadien, auf die Beeinflussung endogener

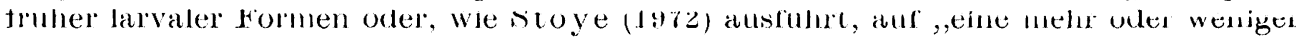
lang andauernde Schädigung des Reproduktionsvermögens der Parasiten im 'Tier" anzusehen ist, läßt sich bei dieser Versuchsanstellung nicht sagen. Ziel der Arbeit war es zu prüfen, inwieweit mit Mebendazol bei der jährlich notwendigen Entwurmung der \%ootiere eine Reduktion der Ausscheidung parasitärer Keime, damit eine Einschränkung der Reinfektionsmöglichkeiten und Absenkung der Wurmbïrde, erreicht werden kann, insbesondere im Hinblick auf eine erfolgreiche Nach- und Aufzucht. Die ausgezeichneten Erfolge sind nicht zuletzt auf die hohe Verträglichkeit des Mittels und seine zögerungsfreie und vollständige Aufnahme durch die Equiden zuriickzuführen.

Die Entwurmungsergebnisse bei Wiederkäuern und Equiden mit Mebendazol der Jahre 1973 und 1974 sind in den Tabellen 1 bis 3 zusammengefaßt.

Der Einsatz von Mebendazol bei Zoowiederkäuern unterscheidet sich gegenüber der Verabreichung an Pferdeartige durch die Herdenfütterung und Kontrolle des Behandlungserfolges ïber Sammelkotproben. Zunächst muß nochmals darauf hingewiesen wer- 
den, daß nicht alle Tiere einer Herde gleichermaßen. aus wolchen (irinden auch immer. Kraftfutter, und damit das Wurmmittel, aufnehmen. Deshalh erfolgte die Verabreichung ïber einen längeren /eitraum mit unterschwelligen Dosen. Damit sollte auch bei unregelmäßiger Annahme eine ausreichende Versorgung mit dem Medikament erreicht werden. Nun kann aber aus betriebstechnischen Griinden nicht für jede Wiederkäuerart. eine eigene Medizinalfuttermischung mit nach Menge verschiedenen Wurmmittelanteilen hergestellt und verfüttert werden. Man ist deshalb bei der Festlegung der Dosis auf den mittleren Kraftfutterverbrauch der verschiedenartigsten Wiederkäuer, deren Körpergewicht zudem durch Schätzung erfaßt wird, angewiesen. Schon die Tiere einer Art fressen nicht proportional ihres Gewichts und noch größere Unterschiede treten von Herde zu Herde auf. Durch Feststellung der taisächlich verbrauchten Kraftfuttermenge einer Tiergruppe und unter Zugrundelegung einer Dosierung von $\overline{\mathrm{j} g} / \mathrm{kg} \mathrm{KGW}$ pro die zeigte sich die zum 'T'eil stark unterschiedliche Versorgung der einzelnen Herden mit dem Anthelminthikum. So nahmen beispielsweise europäische und englische Rothirsche, Rentiere sowie Wapitis lediglich um $3 \mathrm{mg}$. Ture. Mufflons, Nubische Steinböcke und Mähnenschafe dagegen um $8 \mathrm{mg} / \mathrm{kg}$ des Wurmmittels auf. Wie alle unsere Beobachtun-

Tabelle 1. Wurmei- und -larvenausscheidung der Cavicornier vor und nach 14 tägiger Mebendazolverabreichung im Kraftfutter $(620 \mathrm{ppm} ; 5 \mathrm{mg} / \mathrm{kg} \mathrm{KGW} \mathrm{pro}$ (lie)

\begin{tabular}{|c|c|c|c|c|c|c|c|c|}
\hline & \multicolumn{8}{|c|}{ Promentsatz positiver sammelkotproben } \\
\hline & vor & & und & & na & & & \\
\hline & \multicolumn{8}{|c|}{ Mebenclazolangebot } \\
\hline & 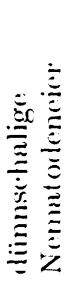 & 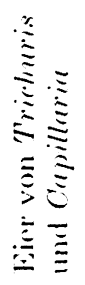 & 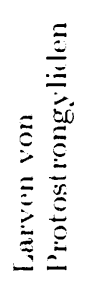 & 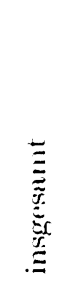 & 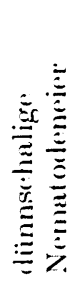 & $\begin{array}{l}\cdots \\
\vdots \\
\vdots \vdots \\
\vdots \vdots \\
\vdots \vdots \\
\vdots \vdots \\
\vdots \\
\vdots \\
\vdots \\
\vdots \\
\vdots\end{array}$ & 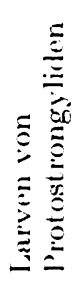 & 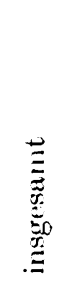 \\
\hline Gemsen & $5 \pi$ & 39 & 39 & $s: 3$ & & & & 0 \\
\hline Nubische steinböcke & 27 & 0 & 60 & 67 & & & & 0 \\
\hline Sibirische Steinböcke & $i$ & $3: 3$ & 20 & 60 & & & & 0 \\
\hline Alpensteinböcke & 18 & 24 & 59 & 82 & & & & 0 \\
\hline 'Ture & 0 & 20 & 47 & 60 & & & & 0 \\
\hline Tahre & 7 & 7 & $1: 3$ & 20 & & & & 0 \\
\hline Markhore & 0 & 29 & 57 & 64 & & & & 0 \\
\hline Mufflons & 68 & 32 & 9 & 77 & & & & 0 \\
\hline Kreishornschafe & 75 & 81 & 94 & 100 & & & & - \\
\hline Mähnenschafe & 7 & 0 & 13 & 20 & & & & 0 \\
\hline Argalis & 50 & 63 & 63 & 88 & & & & - \\
\hline Springböcke & 33 & 0 & 17 & 33 & & & & 0 \\
\hline Hirschziegenantilopen & 100 & 0 & 0 & 100 & & & & 0 \\
\hline Nilgauantilopen & 63 & 13 & 0 & $6: 3$ & & & & 0 \\
\hline Kropfgazellen & 80 & 60 & 0 & 100 & & & & 0 \\
\hline Dorkasgazellen & 67 & 0 & 67 & $8: 3$ & 0 & 0 & 100 & 100 \\
\hline Cavicornier insgesamt & 36 & 27 & 37 & 68 & 0 & 0 & 3 & 3 \\
\hline
\end{tabular}


gen ergahen, waren diese Differenzen nicht durch die Vebendazolzumischung gegeben, sondern entsprachen dem natiurlichen Kraftfutterbedarf. Während bei Equiden die Wurmeiausscheidung auf die angegebene Art vollständig zum Erliegen kam, soll durch weitere Intersuchungen geklärt werden, ob durch Erhöhung der Wurmmittelzugabe, dies ist durch die große toxische Toleranz des Mittels vertretbar, die Lnterdosierung fiir manche Wiederkäuerarten ausgeglichen werden kann. J)aß dann jedoch empfindliche Tiergruppen das medikierte Futter unter Umständen verweigern, wäre möglich. Ähnliches teilen Man\% und Dingeldein (1974) mit, allerdings nach Anwendung eines Anthelminthikums anderer Wirkst offzusammensetzung.

Tabelle 2. Wumei- und -larvenausscheidung der Cerviden vor und nach l4tägiger Mebendazolverabreichung mit dem Kraftfutter $(620 \mathrm{ppm} ; 5 \mathrm{mg} / \mathrm{kg} \mathrm{KGW}$ pro die)

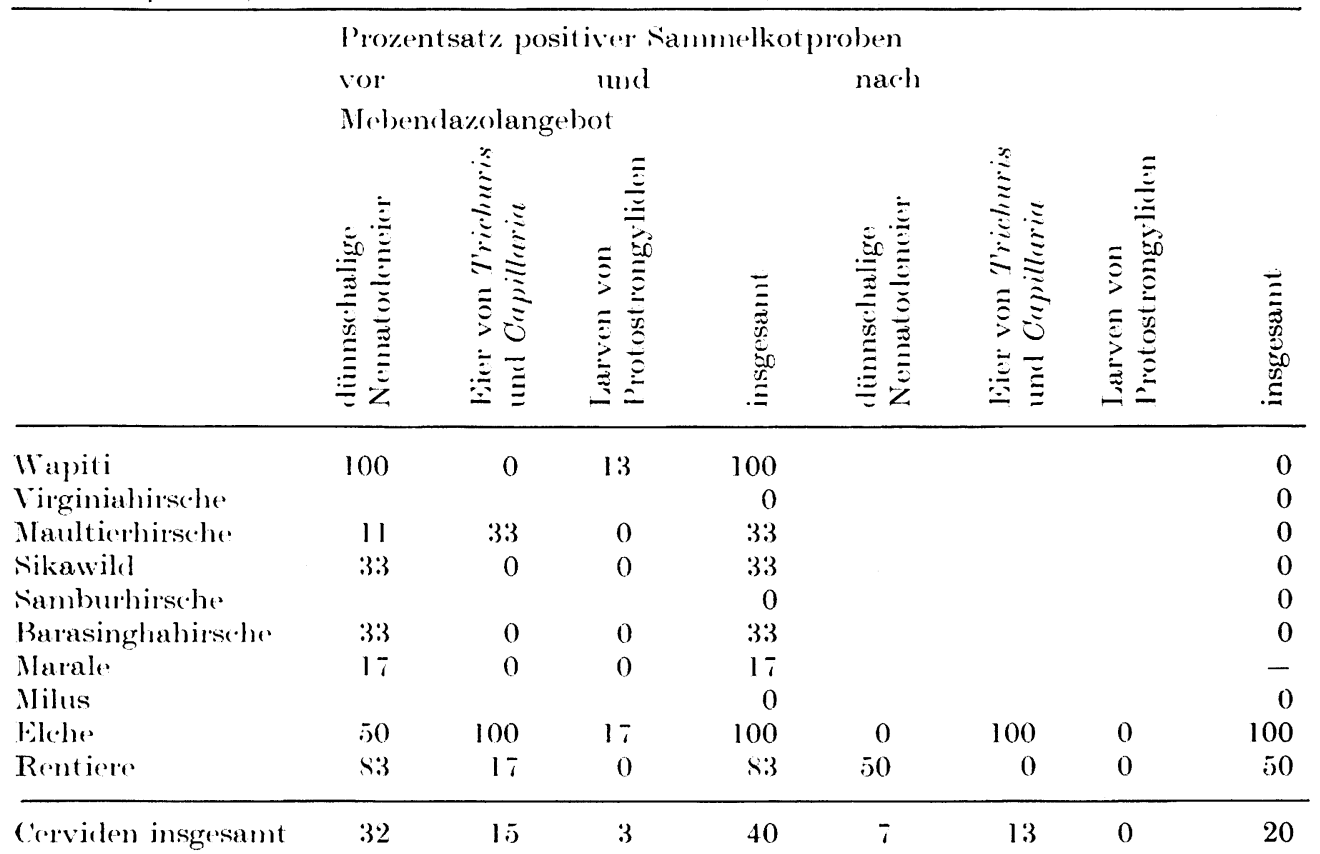

Tabelle 3. Wurmeiausscheidung der Equiden vor und nach 14tägiger Mebendazolverabreichung im Kraftfutter $(400 \mathrm{ppm} ; 1 \mathrm{mg} / \mathrm{kg} \mathrm{KGW}$ pro die)

\begin{tabular}{|c|c|c|c|c|c|c|}
\hline & $\begin{array}{l}\text { Prozents } \\
\text { vor } \\
\text { Mebenda }\end{array}$ & positiver & Einzelkotpr & $\begin{array}{l}\text { oben } \\
\text { nach }\end{array}$ & & \\
\hline & $\begin{array}{l}\text { Strongy- } \\
\text { lideneier }\end{array}$ & $\begin{array}{l}\text { Parascaris- } \\
\text { Eier }\end{array}$ & insgesamt & $\begin{array}{l}\text { Strongy- } \\
\text { lideneier }\end{array}$ & $\begin{array}{l}\text { Parascaris- } \\
\text { Eier }\end{array}$ & insgesamt \\
\hline Zebras & 20 & 41 & 56 & & & 0 \\
\hline Tarpane & 63 & 1 & 64 & & & 0 \\
\hline Przewalskipferde & 88 & 1 & 88 & & & 0 \\
\hline Kulane & & & 0 & & & 0 \\
\hline
\end{tabular}

Equiden insgesamt 44

20

59

0


Zusammenfassend läßt sich aufgrund von dreijährigen Untersuehungen feststellen, daß eine $14 t$ ägige Mebendazolserabreichumg im Kraftetter in täglichen Dosen von $5 \mathrm{mg} / \mathrm{kg}$

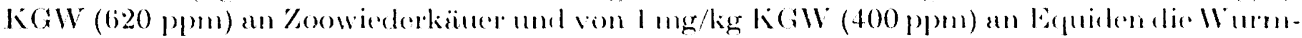
ei- bzw. -larvenausscheidung völlig zu unterelriicken vermag. Mhelentazol warde von allen Tieren gut angenommen unil vertragen.

31 von 34 Wiederkämerherelen (24 (avieornier- und 10 ('ervidengruppen) lieben vor der Behandlung aufgrund der Kotunterste-hung einen Befall mit Magen-1)armst rongy liden (nicht Nematodirus), mehrere mit P'eitschenwïmern und einge mit ('apilharion, 17 Herden mit kleinen Lungenwärmen erkennen. Sitets frei von parasitären Entwicklungsstalien waren clie Proben von Milus sowie Sambur- und Virginiahisthen. Bei den Jikchen crschionen auch nach der Therapie regelmäßig T'richuris- Fier und bei den Dorkasgazellen vereinzelt Larven kleiner Lungenwürmer (Cystocemlus) im kot, wobei jerloch letztere das 11 urmmittel infolge ihree Freßgewohnheiten in zu geringer Menge aufnahmen. - Die P'robleme (ler Diagnosestellung durch Sammelkotproben und der Herdenbehandlung wurden diskutiert.

Von 62 Equiden aus 4 Herden (Zebras, Tarpane, Przewalskipferde und Halbesel) schierlen, wie Untersuchungen am Einzeltier zeigten, 46 Wurmeier aus und zwar 41 von groben und kleinen Strongyliden und 15 von Spulwürmern. Im Jahr 1972 beherbergten 6 von 7 Kulanen Holminthen, die nach der Behandlung, wie auch bei sämtlichen Überprüfungen 1973 nicht mehr nachgewiesen werden konnten.

Mebendazol eignet sich zur Kontrolle des Wurmbefalls bei Wiederkäuern und Pferdeartigen in Tiergärten ausgezeichnet. Seine vielseitige anthelminthische Wirking, seine geringe Toxizität und gute Verträglichkeit versprechen bei systematischer Anwendung eine weitgehende Wurmarmut des Tierbestandes. Damit leistet das Medikament einen wesentlichen Beitrag жur Gesunderhaltung der Zootiere.

\section{Summary}

Examinations during 3 years proved, that the application of Mebendazole together with concentrated foed for 14 days in daily dosis of $5 \mathrm{mg} / \mathrm{kg}$ BW $(620 \mathrm{ppm})$ for ruminants and of $1 \mathrm{mg} / \mathrm{kg} \mathrm{BW}(400 \mathrm{ppm})$ for equides are able to supress completely the elimination of wormeggs respectively -larvae. Mebenda\%ole was well accepted and tolerated by all animals.

31 from 34 flocks of ruminants (24 groups of Crevicomia and 10 of Cerridec) showed by koproscopic examinations before therapy to be infected with intestinal strongyles (not ${ }^{\circ} \%$ matodirus), several with Trichuris and some with Capillaria, 17 with protost rongrle lungworms. The samples from Milus, Sambur and Virginia deer were always free of parasitic stages. After therapy elks eliminated regularly eggs of T'richuris and (icezellu doreres single larvae of small lungworms (Cystocaulus), at which the latters took up the anthelmintic in too small quantities, due to their feeding habits. - The problems of diagnosis by collective samples and of herd therapy were discussed.

From 62 equides of 4 herds (zebras, Equus caballus !melini, E.r. prepralskii, E. hemiomus) on 46 were found wormeggs in the faeces, 41 from large and small strongyles and $15 \mathrm{from}$ Parascaris equorum, as resulted from individual examinations. In 19726 of 7 Equus hemiomus accomodated helminths which could never be detected after therapy and at all investigations during 1973.

To control the attack of worms of ruminants and equides in zoological gardens Mebentazole is highly qualified. His attributes, the wide anthelmintic efficacy, low toxicity and great compatibility, systematically applicated, promise an extensive decrease of helminths in the animal stocks. Therefore the vermifuge provides an essential contribution to the maintenance of healthy zoo animals.

\section{Schrift tum}

Bennett, D. G. (1973): Efficacy of mebendazole as an anthelmintic in horses. Vet. Med./ Small anim. Clin. 68, 604/60 $/ / 609$.

-, -, Bickford, A. A., and Lund, J. E. (1974): Safety evaluation of mebendazole in horses. Am. J. vet. Res. 35, $1003-1004$.

Boch, J. (1961): Phenothiazin, Piperazin und Bephenium als Anthelminthika bei Zootieren. Verhandlungsber. III. Int. Symp. Erkrank. Zootiere, Köln. 


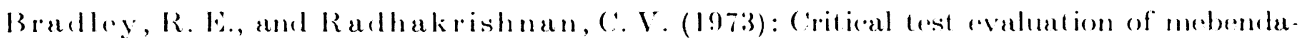
\%ole ugainst gastrointestinal parasites of horses and ponies. Amer. J. vel. Ros. 34, 475) to 477 .

Brgle\%, J., and sienk, L. (1969): Contribution to the knowledge of pathogenieity of trichuriasis in will ruminunts in eaptivity. Verhandlungsber. Xl. Int. Nymp. Frkrank. \%ootiere, Zagreb, $187-188$.

1)ollinger, I'. (1973): \%ur Wirksamkeit des Anthelminlhikums Mebendazol bei \%ootioren. Ibicl. XV. Int. Fymp. Erkrank. \%ootiere, Kolmarlen, 32:3-33:2.

1) uncan, J. L. (1974): Anthelmintic treatment of strongylosis in horses - aclvantages and drawbacks of drugs at present available. ICOD'A III, P'roc. Vol. 3, 14:31 - 14:32.

Encke, W. (I96(i): Desinfektion von Sandgehegen. Vorhandlungsber. VIII. Int. Symp. Erkrank. Zootiere, Leiprig, $8: 3-85$.

Enigk, K. (I96l): Die Bekämpfung der parasitären Infektionen der \%ootiere. Ibid. III. Int. Symp. Erkrank. Zootiere, Köln.

-, -, Dey-Hazra, A.. u. Batke, J. (1973): Zu Wirksamkeit von Mebendazol bei Helminthosen von Huhn und Gans. Avian l'ath. $2,67-74$.

-, -, u. Eckert, J. (1960): Die Desinfektion von Tierausläufen. Zbl. Bakt., I. Abt. Orig. $179,397-432$.

-, -, u. Hilbrich, P. (1968): Die Stallesinfektion bei Parasitosen. 1)tsch. tierärztl. Wschr. 75, $488-492$.

Forstner, M.J. (1967): Untersuchungen über die jahreszeitlich verschicdenen Ausscheidungsraten von Wurmeiern und -larven bei zootieren. Verhandlungsber. IX. Int. Symp. Erkrank. Zootiere, Prag, 167-169.

_, - (1974): P'arasitenbekämpfung bein Hund. Berl. Münch. tierärtl. Wschr. 87, 228-231.

Grevel, V., u. Fckert,J. (1973): Untersuchungen ïber Mebendazol, ein neues Breitspektrumanthelminthikum für die Kleintierpraxis. Schweiz. Arch. Tierheilk. 115, 559-578.

Guilhon, J., Couradeau, G.. et Barnabe, R. (1972b): Action du mébenclazole sur les strongylidés du tube digestif cles equidés. Bull. Acad. vét. Fr. 4.), 331 - :336.

-..,-, Gaillier, 12. et Hubert, J. (1972 a): Action d'un nouveau dérivé de l'imilazole sur les nematodes parasites du tube digestif du mouton. Ibid. 45, 445-452.

Hohenester, H. (1973): Parasitäre Helminthen der Totenkopfaffen (simimiri spp.) mit Untersuchungen über Vorkommen und Bekümpfungsmöglichkeiten. Vet. med. Diss. Miinchen.

Ippen, R., u. Schröder, H.-1). (1970): Über die Verhuste bei der Aufzucht von Süugetieren in zoologischen Gürten. Verhandlungsber. Xll. Int. Symp. Erkrank. \%ootiere, Budapest, $5-13$.

Jan isch, M., u. Varga, I. (1974): Fffica(Y of mebenclazole against gastro-intestinal nematodes in sheep. I(')!'A !II, Proc. Vo!. 3, 1414.

Kaster, H.-1). (1974): Ein Beitrag zur Morphologie exogener Stadien einiger Magen-Darmnematoden von außereuropäischen Fquiden und Sichweineverwandten des Münchner Tierparks Hellabrunn. Vet. mel. Diss. Mïnchen.

Kates, K. C., Golglazier, M. L., Enzie, F. I), Lindahl, I. L., and Samuelson, G. (1974): Helminth control in grazing sheep: Periodic treatment with Levamisole, Morantel, Cambendazole and Mebendazole. J. Parasit. 60, 989-995.

Krüger, W. (1966): Ein Beitrag zur Morphologie ektogener Stadien einiger Magen-Darmnematoden von außereuropäischen Wiederkäuern des Tierparks Hellabrunn in München. Vet. med. Diss. München.

Krumbacher, K. (1965): Helminthologische Untersuchungen an \%ootieren (Pferdeartige, Schweine, Elefanten, Tapire) des Münchner Tierparks Hellabrunn unter besonderer Berücksichtigung der Wurmeiausscheidung im Jahreszyklus. Vet. med. Diss. München.

Kutzer, E., Prosl, H., u. Frey, H. (1974): Zur anthelminthischen Wirkung von Mebendazole (R 17635) beim wildlebenden Wiederkäuer. Dtsch. tierärztl. Wschr. 81, 112-119.

Lahde, G. (1972): Die Bedeutung parasitologischer Kontrolluntersuchungen sowie prophylaktischer und therapeutischer Maßnahmen bei der Bekämpfung der Parasitosen in zoologischen Gärten. Ibid. 7!), 31:3-315. 


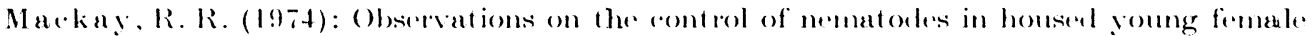
shere. Vet. Rer. $94,73-77$.

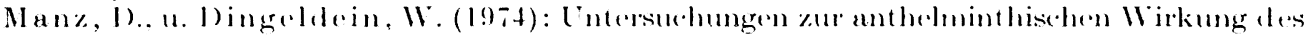

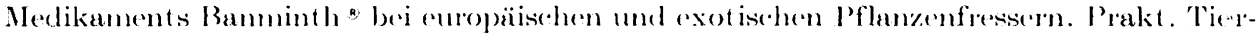
arzt $5 \bar{i}, 422-425$.

Marsboom, R. (I973): Toxicologic: sturlies on mebendazole. Toxieol. appl. Pharmacol. 24 , $371-37 \%$.

Meier, H. W. (1974): (Yber die Wirksankeit des Anthehninthikums Mebendazol (R $17635 \overline{5})$ bei Wildtieren. Prakt. Tierarzt 5.5, $6: 30-633$ s.

Mendheim, H., u. Steinbacher, G. (1956): Cber den Wmmbefall bei Zootieren und seine Behandlung. D. Zool. Garten (NF) $21,263-270$.

Neave, R. M. S., and ('allear, J. F. F. (1973): Further clinical studies on the uses of mebendazole ( $R 17635)$ as an anthelnintic in horses. Brit. vet. J. 12!), $79-82$.

Neunhoeffer, 1. (1965): Untersuchungen über den Wumbefall bei Finhufern und Wiederkäucrn der ,Wilhelma" in stuttgart-Barl Cannstatt unter besonrlerer Beriucksichtigung der jahreszeitlichen Schwankungen in der \%ahl der ausgeschiedenen Wurmeier und -larven. Vet. ned. Diss. München.

Nitz, K. J. (1974): Die anthelminthische Wirkung von Mebendazole als Medizinalfutter bei Schweinen. Tierärztl. Umsch. ㅃ9), 495-496.

Ostmann, O. W. (1973): Telmin-a new equine anthelnintic. Pract. Veterinarian 45, 2:3-25.

Reinecke, R.K., u. Le Roux, D.J. (1972): Anthelmintic activity of mebendazole in equines. J.s. Afr. vet. med. Ass. 43, $287-294$.

Rosicky, B., Erhardova-Kotrla, B. u. (Cerny, V. (1967): Die Finschleppung von \%ooparasiten in zoologische Gärten. Verhandlungsber. IX. Int. Symp. Erkrank. Zootiere, Prag, $161-165$.

Saupe, F., u. Nit\%, K.J. (1972): Zur anthelminthischen Wirkung von Mebenclazole - ein Feldversuch an I'ferden. Berl. Nünch. tieräıtl. Wschr. 85, $21-24$.

Schätzle, M. (1964): Koprologische Untersuchungen bei Wiederkäuern im Münchner Tierpark Hellabrunn unter besonderer Bericksichtigung der jahreszeitlichen Schwankungen der Wurmei- und Wumlarvenausscheidung. Vet. med. Diss. Miunchen.

Scheel, G. (1967): Ein Beitrag zur Therapie des Fncloparasitenbefalles einiger Zoowiederkäuor und equiden. Vet. med. I)iss. München.

Sommer, W. (1975): Beliämpfung des Helninthenbefalles bein P'ferd. Prakt. Tieraryt j(6, $141-142$.

Stoye, M. (1972): P'arasitenbekämpfung beim Pferd. Schweiz. Ar(h. Tierheilk. 114, 601 -613.

Van den Bossche, H. (1972): Biochemical effects of the anthelmintic drug mebendazole. Comp. Biochem. parasit. 139-15\%.

-, - u. Nollin, S. 10 (1973): Fffects of mebenclazole on the absorption of low molecular weight nutrients by Ascroris summ. Int. J. J'arasitol. 3, 401-407.

Walker, l), and Knight, I). (1972): The anthelmintic activity of 'Mebenclazole': A field trial in horses. Vet. Rec. (9), $58-65$.

Wiesner, H. (1973): Zur Behandlung der Nematodeninvasionen von Wassergeflügel mit Mebenclazole (R 17635). Tieräl"ztl. Umsch. 뽀, 135-138.

Zuchowska, F. (1969): P'arasitäre Frkrankungen bei /ebras. Verhandlungsber. XI. Int. Symp. Frkrank. Zootiere, Zagreb, $181-182$.

Prof. 1)r. Dr. h.c. Max Joachim Forstner und Dr. Wilhelm Kraneburg, Institut fiur Vergleichende Tropenmedizin und Parasitologie,

D-8 München 22 (BRD), KÉaulbachstraße 37

Dr. Hen $n$ in $g$ Wiesner,

Münchner Tierpark Hellabrunn,

D-8 Müchen 90 (BRD), Siebenbrunnerstraße 6

Dr. Det lef Jonas,

Landesveterinäruntersuchungsamt Rheinland-Pfalz,

D-54 Koblenz (BRD), Blïcherstraße 34 